\title{
Rigorous, Multimode Equivalent Network Representation of Capacitive Discontinuities
}

\author{
Marco Guglielmi and Alejandro Alvarez Melcón
}

\begin{abstract}
In this paper we present novel, rigorous, multimode equivalent network representations for a variety of zero-thickness capacitive windows and obstacles in a parallel plate waveguide. $A$ key feature of these representations is that the coupling between all of the modes excited is described by a matrix whose elements do not depend on frequency or absolute dimensions. The value of the results presented is that the networks developed can be used to analyze rigorously a large variety of single and coupled planar transmission line structures.
\end{abstract}

\section{INTRODUCTION}

$\mathbf{S}^{\mathrm{E}}$ EVERAL equivalent network representations for capacitive discontinuities are available in the technical literature in terms of impedance (or admittance) equivalent networks ([1], Ch. 6.1.5) [2]-[3]. The equivalent network representations are very useful, when they can be used, because they are extremely efficient from a computational point of view. Most of the network representations that are available, however, only involve fundamental modes and thus have very limited validity.

In a multimode situation, it would be nice to be able to retain the computational efficiency of the impedance (or admittance) networks and still perform a full-wave analysis. The results given in [3] do allow the development of a true multimode equivalent network, but a general network form is not given and the applications discussed are limited to cases in which only the first higher-order mode is explicitly included. Recently, we derived a set of multimode equivalent network representations for inductive discontinuities [4]. The networks derived were rigorous, could be applied to arbitrary geometries, and could easily account for an arbitrary number of higher-order modes.

In this paper, the procedure used in [4] is suitably modified and applied to the analysis of zero-thickness capacitive obstacles and windows in a parallel-plate waveguide. The key component of the network representations derived is an impedance (or admittance) multimode coupling matrix whose elements are obtained in terms of the solution of an integral equation. The solution of this equation is shown to be known so that rigorous expressions for the matrix elements are derived.

Manuscript received July 1, 1992; revised October 23, 1992.

The authors are with the European Space Research and Technology Centre, Noordwijk, The Netherlands.

IEEE Log Number 9209343.
A salient feature of this approach is that the integral equations derived, and therefore also the coupling matrices, do not depend on frequency or absolute dimensions. The computations of the coupling matrix elements thus only involve geometrical parameters that need to be computed only once for each given geometry. The analysis of the structures over a specific frequency range is then carried out via simple network computations that can be performed very rapidly even on small computers.

In this paper, the electromagnetic formulation of the problem is discussed together with the mathematical procedure that leads to the final expressions of the coupling matrix elements. The discontinuities analyzed in the paper are the single and double strip, and the single and double aperture, as shown in Fig. 1. However, the same procedure can be used to obtain network representations for an arbitrary number of strips or apertures as well. Numerical results are presented comparing the results of computations carried out using the networks developed in this paper and the ones obtained using the networks available in the technical literature. The convergence properties of the solutions developed are also discussed indicating how, in addition to being very accurate, the networks developed are also very rapidly convergent.

The value of the results presented in this paper is in that they can be used to develop very efficient codes for the fullwave analysis of a large variety of single and coupled planar transmission line structures.
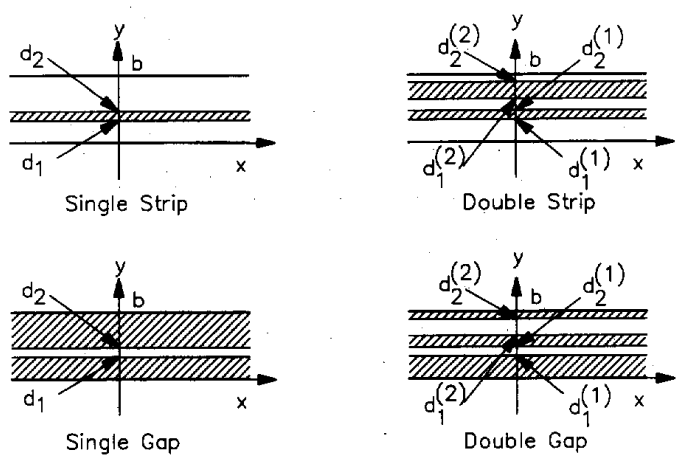

Fig. 1. Metallic, zero-thickness capacitive discontinuities for which rigorous multimode equivalent network representations are derived.

0018-9480/93\$03.00 ㄷ 1993 IEEE 


\section{SINGLE STRIP}

To set up a multimode equivalent network representation for the single strip case, we recognize that since the discontinuity is uniform in the $x$ direction of Fig. 1, to solve the problem we only need to study the discontinuity with the excitation at normal incidence $\left(k_{x}=0\right)$. Once a network representation is derived, the result for the general case with $k_{x} \neq 0$ can be easily obtained by following the procedure outlined in [5]. The excitation is chosen to be $T M$ with respect to $z$ so that only $T M_{m}$ modes will be excited. The relevant vector mode functions can be found in [6], and the characteristic modal impedance is given by

$$
Z_{m}^{(n)}=\frac{\sqrt{k_{o}^{2} \epsilon_{r}^{(n)}-(m \pi / b)^{2}}}{\omega \epsilon_{o} \epsilon_{r}^{(n)}}
$$

where the superscript $(n)$ indicates $z \leq 0$ or $z \geq 0$ for $n=1$ or $n=2$, respectively. It is important to note at this stage that, for $m \rightarrow \infty$, the modal characteristic impedance takes the form

$$
\lim _{m \rightarrow \infty} Z_{m}^{(n)}=\frac{-j(m \pi / b)}{\omega \epsilon_{o} \epsilon_{r}^{(n)}} .
$$

The first step of the formulation is the expansion of the total transverse electric field in terms of the modes of the parallel plate waveguide, obtaining

$$
\boldsymbol{E}_{t}^{(n)}=\sum_{m=0}^{\infty} V_{m}^{(n)}(z) \boldsymbol{e}_{m}(y) .
$$

The next step is the imposition of the boundary conditions on the metal strip, namely,

$$
\sum_{m=0}^{\infty} V_{m}^{(n)} e_{m}(y)=0 ; \quad z=0 .
$$

Let us now observe the behavior of the terms being summed in (4) for $m \rightarrow \infty$, namely,

$$
\begin{aligned}
\lim _{m \rightarrow \infty} V_{m}^{(n)} e_{m}(y) & =\lim _{m \rightarrow \infty}(-1)^{n} I_{m}^{(n)} Z_{m}^{(n)} e_{m}(y) \\
& =(-1)^{n} I_{m}^{(n)} \frac{m B}{\epsilon_{r}^{(n)}} e_{m}(y)
\end{aligned}
$$

where constant $B$ is given by

$$
B=\frac{-j \pi}{b \omega \epsilon_{o}}
$$

and the minus sign on the right-hand side of (5) is a consequence of the assumption that the excitation consists of an arbitrary but finite set of $T M$ modes so that, for $m \rightarrow \infty$, only reflected contributions are present.

As will be evident later, it is now convenient to add to and subtract from (4) the term

$$
-\sum_{m=1}^{\infty} I_{m}^{(1)} \frac{m B}{\epsilon_{r}^{(1)}} \boldsymbol{e}_{m}(y)+\sum_{m=1}^{\infty} I_{m}^{(2)} \frac{m B}{\epsilon_{r}^{(2)}} e_{m}(y),
$$

then add the resulting equations to each other and obtain, with a few manipulations, the following expression:

$$
\sum_{m=0}^{\infty} \bar{V}_{m} \boldsymbol{e}_{m}(y)=\sum_{m=1}^{\infty} \bar{I}_{m} \frac{m B}{\epsilon_{r}^{(1)}+\epsilon_{r}^{(2)}} \boldsymbol{e}_{m}(y)
$$

where the modal voltage and currents $V_{m}^{(n)}$ and $I_{m}^{(n)}$ have been redefined according to

$$
\begin{aligned}
I_{m}^{(1)}-I_{m}^{(2)} & =\bar{I}_{m} \\
V_{m}+\bar{I}_{m} \frac{m B}{\epsilon_{r}^{(1)} \epsilon_{r}^{(2)}} & =\bar{V}_{m} .
\end{aligned}
$$

The next step in the solution of the problem is the use in (8) of the transform relation between the transverse magnetic field at the discontinuity location and the modal currents, obtaining

$$
\bar{I}_{n}=\int_{d_{1}}^{d_{2}}\left[-z_{o} \times\left(\boldsymbol{H}^{(1)}-\boldsymbol{H}^{(2)}\right)\right] \cdot \boldsymbol{e}_{n}^{*} d y^{\prime} .
$$

The above expression can now be used in (8), obtaining

$$
\begin{aligned}
\sum_{m=0}^{\infty} \bar{V}_{m} \boldsymbol{e}_{m}(y)= & \int_{d_{1}}^{d_{2}}\left[-\boldsymbol{z}_{o} \times\left(\boldsymbol{H}^{(1)}-\boldsymbol{H}^{(2)}\right)\right] \\
& \cdot \frac{B}{\epsilon_{r}^{(1)}+\epsilon_{r}^{(2)}} \sum_{n=1}^{\infty} n \boldsymbol{e}_{n} \boldsymbol{e}_{n}^{*} d y^{\prime}
\end{aligned}
$$

Note that, in writing this last equation, we have changed, for convenience, the index of the summation from $m$ to $n$ in the right-hand side of (8).

Due to the linearity of the problem, we can define a set of unknown functions $M_{m}$ which are related to the transverse magnetic field via

$$
-\boldsymbol{z}_{o} \times\left(\boldsymbol{H}^{(1)}-\boldsymbol{H}^{(2)}\right)=\sum_{m=0}^{\infty} \bar{V}_{m} \boldsymbol{A}_{\circ} M_{m}\left(y^{\prime}\right)
$$

where $\boldsymbol{A}_{o}$ is a vector constant to be defined later.

Inserting (13) in (12), and comparing like coefficients, the following integral equation is finally obtained:

$$
\boldsymbol{e}_{m}=\int_{d_{1}}^{d_{2}} M_{m}\left(y^{\prime}\right) \boldsymbol{A}_{o} \cdot \frac{B}{\epsilon_{r}^{(1)}+\epsilon_{r}^{(2)}} \sum_{n=1}^{\infty} n \boldsymbol{e}_{n} \boldsymbol{e}_{n}^{*} d y^{\prime}
$$

The completion of the network formulation is obtained by substituting expression (13) into (11), finding

$$
\bar{I}_{n}=\sum_{M=0}^{\infty} \bar{V}_{m} \int_{d_{1}}^{d_{2}} A_{o} M_{m}\left(y^{\prime}\right) \cdot e_{n}^{*} d y^{\prime} .
$$

This expression can now be used to define the multimode equivalent network representation in Fig. 2, where the generic element $Y_{n, m}$ of the multimode coupling matrix is given by

$$
Y_{n, m}=\int_{d_{1}}^{d_{2}} M_{m}\left(y^{\prime}\right) A_{o} \cdot e_{n}^{*} d y^{\prime} .
$$

It is important to note that the derivation of the fundamental integral equation has been carried without introducing any approximations. Furthermore, the kernel of (14) is not frequency dependent and this greatly simplifies the solution of the problem. The only frequency dependent term is $B$, as defined in (6). As a consequence, the resulting expression for the generic coupling matrix element $Y_{n, m}$ has the same, very simple frequency dependence.

The last step of the procedure is the solution of (14). As will be shown, this integral equation can be reduced to a Cauchy-type singular integral equation of known solution [7]. 


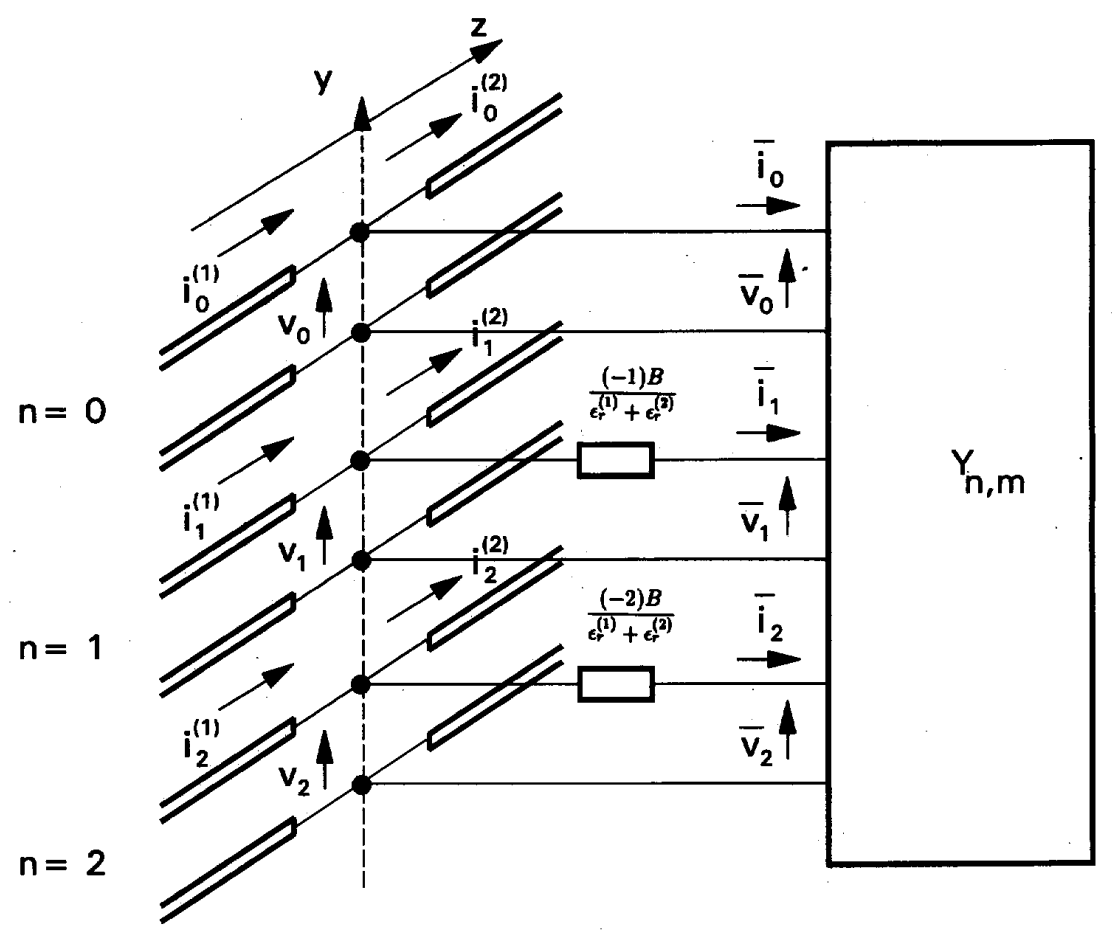

Fig. 2. Specific form of the equivalent network representation of the capacitive single (and double) strip in Fig. 1. For the sake of space, only three modes are shown explicitly.

As a consequence, closed-form analytical expressions for the generic element of the multimode coupling matrix can be easily obtained. The details of the mathematical procedure of the solution of (14) are reported in the Appendix A. The final expression for the generic coupling matrix element is

$$
\begin{aligned}
Y_{n, m}= & \sqrt{\epsilon_{n} \epsilon_{m}} \frac{k_{2}^{2}\left(\epsilon_{r}^{(1)}+\epsilon_{r}^{(2)}\right)}{\pi B} \\
& \cdot \int_{-1}^{1} \frac{R_{n}\left(\nu^{\prime}\right)\left[Q_{m}\left(\nu^{\prime}\right)-C_{m}\right]}{\sqrt{1-\left(k_{1}+k_{2} \nu^{\prime}\right)^{2}} \sqrt{1-\nu^{\prime 2}}} d \nu^{\prime}
\end{aligned}
$$

where

$$
\begin{gathered}
R_{n}(y)=\frac{1}{k_{2}} \begin{cases}\frac{\pi}{b}\left(y-d_{2}\right) ; & n=0 \\
\frac{1}{n}\left[\sin \left(\frac{n \pi}{b} y\right)-\sin \left(\frac{n \pi}{b} d_{2}\right)\right] ; & n \neq 0 \\
y=\frac{b}{\pi} \arccos \left[k_{1}+k_{2} \nu^{\prime}\right]\end{cases} \\
Q_{m}\left(\nu^{\prime}\right)=\frac{1}{\pi} \quad \\
\int_{-1}^{1} \frac{\sqrt{1-\nu^{2}} f_{m}\left(\frac{b}{\pi} \arccos \left[k_{1}+k_{2} \nu\right]\right)}{\nu-\nu^{\prime}} d \nu \\
C_{m}=\frac{1}{D} \int_{-1}^{1} \frac{f_{m}(y)=\cos \left(\frac{m \pi}{b} y\right)}{\sqrt{1-\left(k_{1}+k_{2} \nu\right)^{2}} \sqrt{1-\nu^{2}}} d \nu \\
D=\int_{-1}^{1} \frac{d \nu}{\sqrt{1-\left(k_{1}+k_{2} \nu\right)^{2}} \sqrt{1-\nu^{2}}}
\end{gathered}
$$

$$
\begin{aligned}
k_{n} & =\frac{\eta_{2}-(-1)^{n} \eta_{1}}{2} \\
\eta_{1,2} & =\cos \left(\frac{\pi}{b} d_{1,2}\right) .
\end{aligned}
$$

\section{DOUBLE STRIP}

The formulation of the double-strip problem is essentially identical to the single strip case. The only difference is that now all integrations are performed over an interval that has a gap in it, corresponding to the air gap between the strips in Fig. 1. As a result, the network shown in Fig. 2 is still applicable, provided that the appropriate expression is used for the coupling matrix elements $Y_{n, m}$. Using the single-strip results derived in the previous section, we can therefore write directly

$$
\begin{aligned}
Y_{n, m} & =f_{d_{1}^{(1)}}^{d_{2}^{(2)}} M_{m}\left(y^{\prime}\right) \boldsymbol{A}_{o} \cdot \boldsymbol{e}_{n}^{*} d y^{\prime} \\
\boldsymbol{e}_{m} & =f_{d_{1}^{(1)}}^{d_{2}^{(2)}} M_{m}\left(y^{\prime}\right) \boldsymbol{A}_{o} \cdot \frac{B}{\epsilon_{r}^{(1)}+\epsilon_{r}^{(2)}} \sum_{n=1}^{\infty} n \boldsymbol{e}_{n} \boldsymbol{e}_{n}^{*} d y^{\prime}
\end{aligned}
$$

where the bar through the integrals means that the integration variable cannot assume values in the interval $\left[d_{2}^{(1)}, d_{1}^{(2)}\right]$ in Fig. 1.

Equation (27) can again be reduced to a Cauchy-type singular integral equation of known solution ([1], Ch. 7.1). The mathematical details are reported in Appendix A, and the final expression for the generic coupling matrix element now 
becomes

$$
\begin{aligned}
Y_{n, m}= & \sqrt{\epsilon_{n} \epsilon_{m}} \frac{k_{2}^{2}\left(\epsilon_{r}^{(1)}+\epsilon_{r}^{(2)}\right)}{\pi B} \\
& \cdot \int_{-1}^{1}\left[F_{n, m}\left(k_{1}^{u}+k_{2}^{u} \nu\right) k_{2}^{u}+F_{n, m}\left(k_{1}^{l}+k_{2}^{l} \nu\right) k_{2}^{l}\right] d \nu
\end{aligned}
$$

where

$$
\begin{aligned}
& F_{n, m}(\xi)=\frac{\left\{Q_{m}^{u}(\xi)+Q_{m}^{l}(\xi)-P_{m}(\xi)\right\} R_{n}(\xi)}{X(\xi) \sqrt{1-\left(k_{1}+k_{2} \xi\right)^{2}}} \\
& R_{n}(\xi)=\frac{1}{k_{2}} \begin{cases}\arccos \left(k_{1}+k_{2} \xi^{\prime}\right) t_{1}^{\xi} ; & n=0 \\
\frac{1}{n} \sin \left[n \arccos \left(k_{1}+k_{2} \xi^{\prime}\right)\right] \downarrow_{1}^{\xi} ; & n \neq 0\end{cases} \\
& X(\xi)=\sqrt{1-\xi^{2}}(\eta-1) \sqrt{\frac{\eta+1}{\eta-1}} \\
& \eta=\frac{2}{\eta_{2}^{(1)}-\eta_{1}^{(2)}}\left[\xi-\frac{1}{2}\left(\eta_{2}^{(1)}+\eta_{1}^{(2)}\right)\right] \\
& \eta_{1,2}^{(n)}=\cos \left(\frac{\pi}{a} d_{1,2}^{(n)}\right) \\
& k_{n}=\frac{\eta_{2}^{(2)}-(-1)^{n} \eta_{1}^{(1)}}{2} \\
& \xi_{2,1}^{(1,2)}=\frac{1}{k_{2}}\left[\eta_{2,1}^{(1,2)}-k_{1}\right] \\
& k_{n}^{u}=\frac{1-(-1)^{n} \xi_{1}^{(2)}}{2} \\
& k_{n}^{l}=\frac{\xi_{2}^{(1)}+(-1)^{n}}{2} \\
& P_{m}(\xi)=C_{m}^{0}+C_{m}^{1} \xi \\
& C_{m}^{0}=\frac{T_{m}^{u} S_{1}^{l}-T_{m}^{l} S_{1}^{u}}{S_{0}^{u} S_{1}^{l}-S_{0}^{l} S_{1}^{u}} \\
& C_{m}^{1}=\frac{T_{m}^{l} S_{0}^{u}-T_{m}^{u} S_{0}^{l}}{S_{0}^{u} S_{1}^{l}-S_{0}^{l} S_{1}^{u}} \\
& S_{n}^{u, l}=\int_{-1}^{1}
\end{aligned}
$$

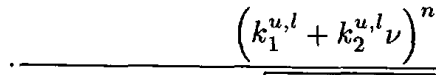

$$
\begin{aligned}
& X\left(k_{1}^{u, l}+k_{2}^{u, l} \nu\right) \sqrt{1-\left[k_{1}+k_{2}\left(k_{1}^{u, l}+k_{2}^{u, l} \nu\right)\right]^{2}} \\
& \cdot k_{2}^{u, l} d \nu
\end{aligned}
$$

$$
\begin{array}{r}
T_{m}^{u, l}=\int_{-1}^{1} \\
\cdot \frac{Q_{m}^{u}\left(k_{1}^{u, l}+k_{2}^{u, l} \nu\right)+Q_{m}^{l}\left(k_{1}^{u, l}+k_{2}^{u, l} \nu\right)}{X\left(k_{1}^{u, l}+k_{2}^{u, l} \nu\right) \sqrt{1-\left[k_{1}+k_{2}\left(k_{1}^{u, l}+k_{2}^{u, l} \nu\right)\right]^{2}}} \\
\cdot k_{2}^{u, l} d \nu
\end{array}
$$

$$
\begin{aligned}
& Q_{m}^{u, l}\left(k_{1}^{u, l}+k_{2}^{u, l} \nu\right)=\frac{1}{\pi} \\
& \cdot \int_{-1}^{1} \frac{X\left(k_{1}^{u, l}+k_{2}^{u, l} \nu^{\prime}\right) f_{m}\left(k_{1}^{u, l}+k_{2}^{u, l} \nu^{\prime}\right)}{\nu^{\prime}-\nu} d \nu^{\prime}
\end{aligned}
$$

$$
\begin{gathered}
Q_{m}^{u, l}\left(k_{1}^{l, u}+k_{2}^{l, u} \nu\right)=\frac{1}{\pi} \\
\int_{-1}^{1} \frac{X\left(k_{1}^{u, l}+k_{2}^{u, l} \nu^{\prime}\right) f_{m}\left(k_{1}^{u, l}+k_{2}^{u, l} \nu^{\prime}\right)}{\left(k_{1}^{l, u}-k_{1}^{l, u}\right)+\left(k_{1}^{u, l} \nu^{\prime}-k_{2}^{l, u} \nu\right)} k_{2}^{\mu, l} d \nu^{\prime} \\
f_{m}(\xi)=\cos \left[m \arccos \left(k_{1}+k_{2} \xi\right)\right] .
\end{gathered}
$$

\section{Single APERTURE}

The multimode equivalent network for the single and double strip cases has been set up in terms of the magnetic field discontinuity due to the present of the metal strip. For the single and double aperture cases, it is more convenient to set up the formulation in terms of the electric field in the air gaps.

The first step in the formulation is again the expansion of the total transverse magnetic field in the form

$$
\boldsymbol{H}_{t}^{(n)}=\sum_{m=0}^{\infty} I_{m}^{(n)}(z) h_{m}(y)
$$

where the superscript $(n)$ indicates $z \leq 0$ or $z \geq 0$, for $n=1$ or $n=2$, respectively. The next step is the imposition of the boundary conditions in the air gap, obtaining

$$
\sum_{m=0}^{\infty} I_{m}^{(1)} \boldsymbol{h}_{m}(y)=\sum_{m=0}^{\infty} I_{m}^{(2)} \boldsymbol{h}_{m}(y) ; \quad z=0 .
$$

Similarly to what was done previously, we now observe the behavior of the terms being summed on the left- and right-hand sides of (47) for $m \rightarrow \infty$, obtaining

$$
\lim _{m \rightarrow \infty} I_{m}^{(n)} h_{m}(y)=(-1)^{n} V_{m}^{(n)} \frac{\epsilon_{r}^{(n)}}{m B} h_{m}(y)
$$

where constant $B$ is again given by (6), and the minus sign on the right-hand side of (48) is again a consequence of the assumption that the excitation consists of a finite set of $T M$ modes so that, for $m \rightarrow \infty$, only reflected contributions are present.

Following the same procedure developed for the single strip case, we now add and subtract to both sides of (47) the term

$$
-\sum_{m=1}^{\infty} V_{m}^{(1)} \frac{\epsilon_{r}^{(1)}}{B m} h_{m}(y)+\sum_{m=1}^{\infty} V_{m}^{(2)} \frac{\epsilon_{r}^{(2)}}{B m} h_{m}(y)
$$

and obtain, with a few manipulations, the following expression:

$$
\sum_{m=0}^{\infty} \bar{I}_{m} \boldsymbol{h}_{m}(y)=\sum_{m=1}^{\infty} \bar{V}_{m} \frac{\left(\epsilon_{r}^{(1)}+\epsilon_{r}^{(2)}\right)}{B m} \boldsymbol{h}_{m}(y)
$$

where the modal voltage and currents $V_{m}^{(n)}$ and $I_{m}^{(n)}$ have been redefined according to

$$
\begin{gathered}
I_{m}^{(1)}-I_{m}^{(2)}+\bar{V}_{m} \frac{\left(\epsilon_{r}^{(1)}+\epsilon_{r}^{(2)}\right)}{B m}=\bar{I}_{m} \\
V_{m}^{(1)}=V_{m}^{(2)}=\bar{V}_{m} .
\end{gathered}
$$


The next step in the solution of the problem is the use in (50) of the transform relation between the transverse electric field and the modal voltage, namely,

$$
\bar{V}_{n}=\int_{d_{1}}^{d_{2}}\left[z_{o} \times E\right] \cdot h_{n}^{*} d y^{\prime}
$$

obtaining, after a few manipulations, the following expression:

$$
\begin{aligned}
\sum_{m=0}^{\infty} \bar{I}_{m} \boldsymbol{h}_{m}(y)= & \int_{d_{1}}^{d_{2}}\left[\boldsymbol{z}_{o} \times \boldsymbol{E}\right] \cdot B^{-1}\left(\epsilon_{r}^{(1)}+\epsilon_{r}^{(2)}\right) \\
& \cdot \sum_{n=1}^{\infty} \frac{\boldsymbol{h}_{n}(y) \boldsymbol{h}_{n}^{*}\left(y^{\prime}\right)}{n} d y^{\prime}
\end{aligned}
$$

(note that, since the obstacle is zero thickness, we have $\boldsymbol{E}^{(1)}=\boldsymbol{E}^{(2)}=\boldsymbol{E}$ in the air gap). In writing this last equation, we have changed, for convenience, the index of the summation from $m$ to $n$ on the right-hand side of (50).

Due to the linearity of the problem, we can again define a set of unknown functions $M_{m}$ which are related to the transverse electric field in the air gap via

$$
\left(z_{o} \times \boldsymbol{E}\right)=\sum_{m=0}^{\infty} \bar{I}_{m} \boldsymbol{A}_{o} M_{m}\left(y^{\prime}\right)
$$

where $A_{o}$ is a vector constant to be defined later.
Inserting now (55) in (54), and comparing like coefficients, the following integral equation is obtained:

$$
\begin{aligned}
h_{m}(y)= & \int_{d 1}^{d 2} M_{m}(y) \boldsymbol{A}_{o} \cdot B^{-1}\left(\epsilon_{r}^{(1)}+\epsilon_{r}^{(2)}\right) \\
& \cdot \sum_{n=1}^{\infty} \frac{\boldsymbol{h}_{n}(y) \boldsymbol{h}_{n}^{*}\left(y^{\prime}\right)}{n} d y^{\prime} .
\end{aligned}
$$

The completion of the network formulation is obtained by substituting expression (55) into (53), finding

$$
\bar{V}_{n}=\sum_{m=0}^{\infty} \bar{I}_{m} \int_{d_{1}}^{d_{2}} \boldsymbol{A}_{o} \boldsymbol{M}_{m}\left(y^{\prime}\right) \boldsymbol{h}_{m}^{*}\left(y^{\prime}\right) d y^{\prime} .
$$

The result of this last step can again be used to define a multimode equivalent network representation (Fig. 3) where the expression for the generic element $Z_{n, m}$ of the multimode coupling matrix is given by

$$
Z_{n, m}=\int_{d 1}^{d 2} M_{m}\left(y^{\prime}\right) A_{o} \cdot h_{n}^{*}\left(y^{\prime}\right) d y^{\prime} .
$$

It is important to note that the integral equation derived has all the features already outlined for the strip case. The details of the mathematical procedure of its solution are reported in Appendix B. The resulting final expression for the generic coupling matrix elements is

$Z_{n, m}=\frac{B \sqrt{\epsilon_{m} \epsilon_{n}}}{\left(\epsilon_{r}^{(1)}+\varepsilon_{r}^{(2)}\right) \pi^{2}}\left[\frac{-D_{0}^{(n)} D_{0}^{(m)}}{\log S}+\sum_{k=1}^{\infty} 2 k D_{k}^{(n)} D_{k}^{(m)}\right]$

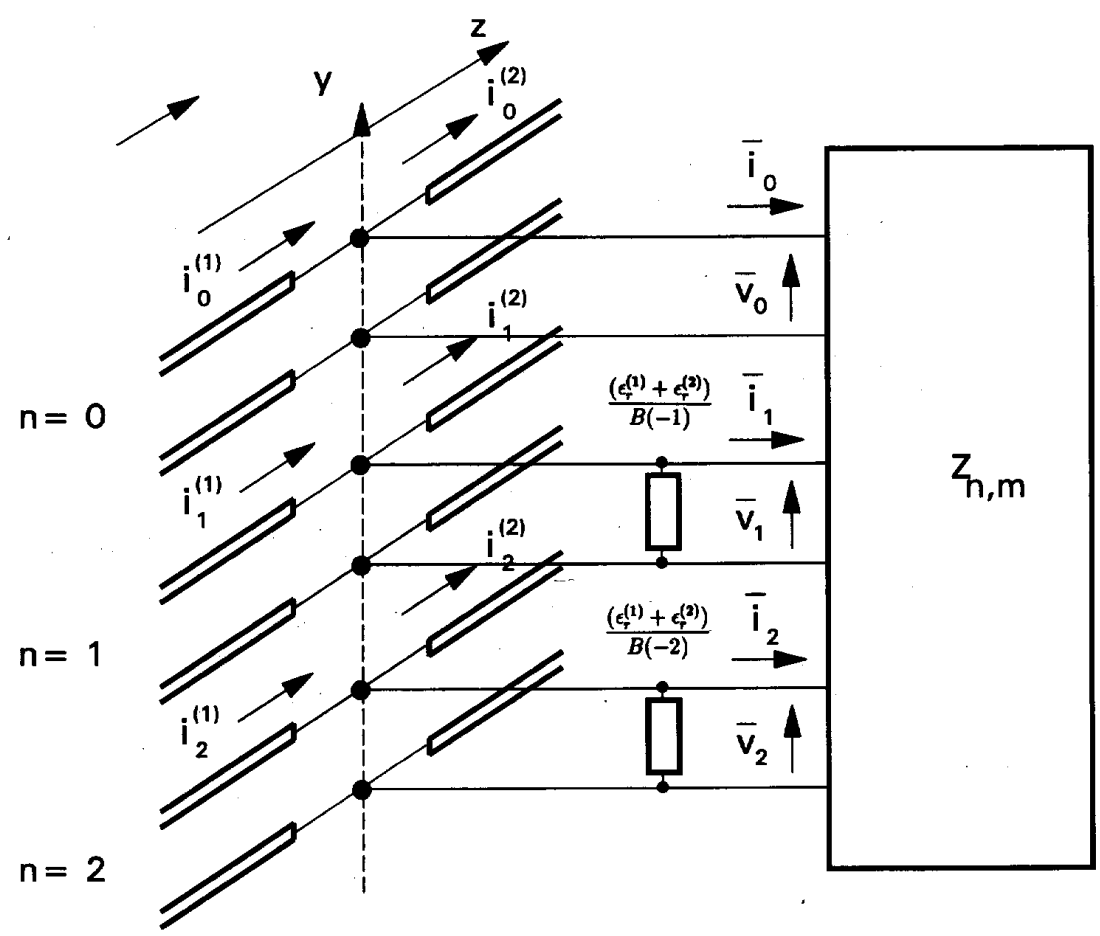

Fig. 3. Specific form of the equivalent network representation of the capacitive single (and double) aperture in Fig. 1. For the sake of space, only three modes are shown explicitly. 
where

$$
\begin{gathered}
D_{k}^{(m)}=\int_{0}^{\pi} \cos (k x) \cos [m \arccos (C+S \cos x)] d x \\
C=\frac{1}{2}\left(\cos \frac{\pi d_{1}}{b}+\cos \frac{\pi d_{2}}{b}\right) \\
S=\frac{1}{2}\left(\cos \frac{\pi d_{1}}{b}-\cos \frac{\pi d_{2}}{b}\right) .
\end{gathered}
$$

\section{DOUBLE APERTURE}

The network representation shown in Fig. 3 can be used for the case of the double capacitive aperture shown in Fig. 1 as well. To obtain the appropriate expression for the coupling matrix element $Z_{n, m}$, we only need to introduce an additional gap in the integration intervals. The fundamental equations then becomes

$$
\begin{gathered}
Z_{n, m}=f_{d_{1}^{(1)}}^{d_{2}^{(2)}} M_{m}\left(y^{\prime}\right) A_{0} \cdot h_{n}^{*}\left(y^{\prime}\right) d y^{\prime} \\
h_{m}=\int_{d_{1}^{(1)}}^{d_{2}^{(2)}} M_{m}\left(y^{\prime}\right) A_{0} \cdot B^{-1}\left(\epsilon_{r}^{(1)}+\epsilon_{r}^{(2)}\right) \\
\cdot \sum_{n=1}^{\infty} \frac{h_{n}(y) h_{n}\left(y^{\prime}\right)}{n} d y^{\prime}
\end{gathered}
$$

where the bar through the integral means that the integration variable cannot assume values in the interval $\left[d_{2}^{(1)}, d_{1}^{(2)}\right]$ in Fig. 1.

Equation (64) can again be solved analytically, and we refer the reader to Appendix B for the mathematical details of its solution. The resulting final expression for the coupling matrix elements is given by

$$
\begin{aligned}
Z_{n, m}=\frac{B \sqrt{\epsilon_{n} \epsilon_{n}}}{\pi^{2}\left(\varepsilon_{r}^{(1)}+\epsilon_{r}^{(2)}\right)}[ & \left(\frac{-1}{\log S}\right)\left(\beta_{0}^{(m)}+\gamma^{(m)}\right) \beta_{0}^{(n)} \\
& \left.+2 \sum_{k=1}^{\infty} k\left(\beta_{k}^{(m)}+I_{k}^{(m)}\right) \beta_{k}^{(n)}\right]
\end{aligned}
$$

where

$$
\begin{gathered}
\beta_{k}^{(n)}=\int_{0}^{\pi} \cos (k x) \cos [n \arccos (C+S \cos x)] d x \\
I_{k}^{(m)}=k_{2}^{2}\left[C_{m} \rho_{k}+\frac{1}{\log S}\left(\beta_{0}^{(m)}+I_{0}^{(m)}\right) \lambda_{k}^{(0)}\right. \\
\left.-2 \sum_{l=1}^{\infty} l \beta_{l}^{(m)} \lambda_{k}^{(l)}\right] \\
C_{m}=\frac{S_{1}^{(m)} R_{2}-S_{2}^{(m)} R_{1}}{\rho_{0} R_{2}-R_{1} \Delta} \\
\gamma^{(m)}=\frac{S_{2}^{(m)} \rho_{0}-S_{1}^{(m)} \Delta}{\rho_{0} R_{2}-R_{1} \Delta} \\
R_{1}=\frac{\lambda_{0}^{(0)}}{\log S}-\frac{1}{k_{2}^{2}} \\
R_{2}=\frac{1}{\log S} \Delta_{0}
\end{gathered}
$$

$$
\begin{aligned}
& S_{1}^{(m)}=\frac{-1}{\log S} \beta_{0}^{(m)} \lambda_{0}^{(0)}+2 \sum_{l=1}^{\infty} l \beta_{l}^{(m)} \lambda_{0}^{(l)} \\
& S_{2}^{(m)}=\frac{-1}{\log S} \beta_{0}^{(m)} \Delta_{0}+2 \sum_{l=1}^{\infty} l \beta_{l}^{(m)} \Delta_{l} \\
& \rho_{k}=\frac{1}{\pi} \int_{-1}^{1} \frac{R_{k}(\xi)}{\sqrt{1-\left(k_{1}+k_{2} \xi\right)^{2}} \sqrt{1-\xi^{2}}} d \xi \\
& \lambda_{k}^{(l)}=\frac{1}{\pi} \int_{-1}^{1} \frac{R_{k}(\xi) Q_{l}(\xi)}{\sqrt{1-\left(k_{1}+k_{2} \xi\right)^{2}} \sqrt{1-\xi^{2}}} d \xi \\
& \Delta=\frac{1}{\pi} \int_{-1}^{1} \frac{1}{\sqrt{1-\left(k_{1}+k_{2} \xi\right)^{2}} \sqrt{1-\xi^{2}}} d \xi \\
& \Delta_{l}=\frac{1}{\pi} \int_{-1}^{1} \frac{Q_{l}(\xi)}{\sqrt{1-\left(k_{1}+k_{2} \xi\right)^{2}} \sqrt{1-\xi^{2}}} d \xi \\
& Q_{l}(\xi)=\frac{1}{\pi} \int_{-1}^{1} \frac{\sqrt{1-\xi^{\prime 2}}}{\xi^{\prime}-\xi} \cos \left[l \arccos \left(k_{1}+k_{2} \xi^{\prime}\right)\right] d \xi^{\prime} \\
& Q_{0}(\xi)=-\xi \\
& R_{k}=\frac{-1}{k_{2}} \begin{cases}\left.\arccos \left(k_{1}+k_{2} \xi^{\prime}\right)\right|_{\xi} ^{1} ; & k=0 \\
\left.\frac{1}{k} \sin \left[k \arccos \left(k_{1}+k_{2} \xi^{\prime}\right)\right]\right|_{\xi} ^{1} ; & k \neq 0\end{cases} \\
& C=\frac{1}{2}\left(\cos \frac{\pi d_{1}^{(1)}}{b}+\cos \frac{\pi d_{2}^{(2)}}{b}\right) \\
& S=\frac{1}{2}\left(\cos \frac{\pi d_{1}^{(1)}}{b}-\cos \frac{\pi d_{2}^{(2)}}{b}\right) \\
& k_{n}=\frac{1}{2}\left(\eta_{2}-(-1)^{n} \eta_{1}\right) \\
& \begin{array}{c}
\eta_{n}=\cos x_{n} \\
x_{1}=\arccos \left[\frac{1}{S}\left(\cos \frac{\pi d_{2}^{(1)}}{b}-C\right)\right]
\end{array} \\
& x_{2}=\arccos \left[\frac{1}{S}\left(\cos \frac{\pi d_{1}^{(2)}}{b}-C\right)\right] \text {. }
\end{aligned}
$$

\section{NuMERICAL CONVERGENCE PROPERTIES}

The expressions derived in the previous sections for the generic coupling matrix elements of the single and double strip cases generally involve integrations that are best performed numerically. In all of the cases that we considered, a 96point Gauss integration procedure has been used [8], for a maximum modal index $m=40$, obtaining very good numerical convergence.

The results developed for the single and double aperture cases contain summations in addition to integrals. The integrals have been treated numerically in the same fashion as for the strip cases without any difficulty. What is left is to evaluate the convergence properties of the summations in (59) and (65). Fig. 4 shows the variation of the value of the coupling matrix element for $n=10$ and $m=10$ according to (59) (single aperture) as a function of the number of terms summed. As we can see, adding ten terms, good convergency has already been achieved. 
The double aperture case is slightly more complex than the single aperture since it involves double summations. Again, in Fig. 4, the convergence behavior of the coupling matrix element for $n=10$ and $m=10$ according to (65) (double aperture) is shown (dotted line). For this calculation, 28 terms have been computed in all the other sums required. The discontinuous behavior in Fig. 4 is due to the fact that the test was performed using a centered obstacle (single or double aperture). In this case the even modes are not excited and, therefore, the contribution presented by these modes is negligible.

We have also evaluated the convergence of the inner summations, and it has been found that the values of $S_{1}^{(m)}$ and $S_{2}^{(m)}$, computed for $m=10$, according to $(72)$ and $(73)$, reach a reasonable constant value with again just 10 elements. As a conclusion, we can say that in spite of the more complex analytical expression for $Z_{n, m}$, the convergence properties of the double aperture are similar to the ones of the single aperture. Furthermore, if the modal index $m$ or $n$ is increased, a similar behavior is observed, obtaining good numerical convergence with a number of terms equal to the largest modal index.

An additional convergence test that is useful in appreciating the value of the representations derived is its modal convergency, namely, the number of modes that must be explicitly included in order to obtain the numerical convergency of the results obtained from the network calculations. In Fig. 5 we show the normalized susceptance introduced by a centered capacitive strip, and by a centered capacitive aperture in the lowest mode of a rectangular waveguide, versus the number of higher order modes explicitly included in the calculations. As we can see, in both of the cases, ten modes are enough to obtain acceptable numerical accuracy.

In the example shown in Fig. 5 , only one mode is above cutoff in the waveguide. If the frequency of operation is increased, so that more modes are allowed to propagate, one must increase the number of modes explicitly included in the network calculations. The exact number of modes that must

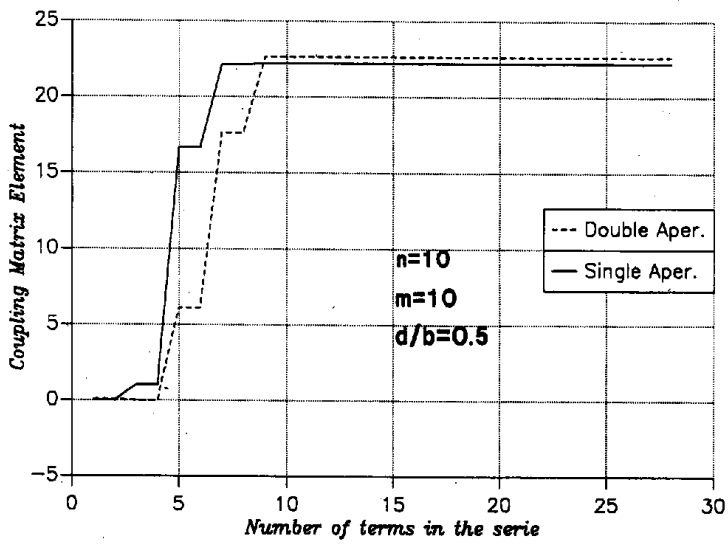

Fig. 4. Convergence behavior of the coupling matrix elements for the single and double aperture problem. The computed parameter is $Z_{n, m}$ in (59) and (65) for $n=10$ and $m=10$.

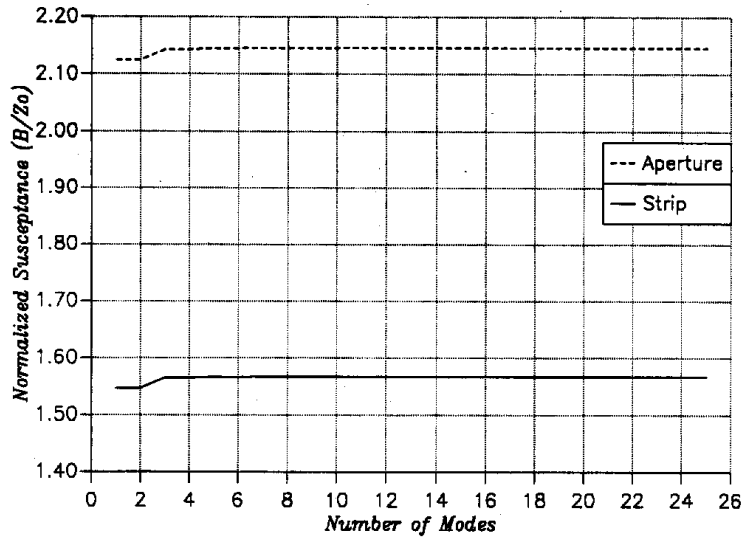

Fig. 5. Number of modes needed to get numerical convergency with the network developed. The computed parameter is the normalized susceptance introduced by a centered capacitive strip and by a centered capacitive aperture in the lowest mode of a rectangular waveguide. The waveguide dimensions are those in Fig. 4 with $d / b=0.9$ for the capacitive strip and $d / b=0.05$ for the capacitive gap.

be included for a specific structure is best determined by observing the numerical convergence of the electrical parameter of interest. Note, however, that the network representations derived do not exhibit any relative convergence phenomenon, but give uniformly convergent results as the number of modes is increased.

\section{VALIDATION OF THE RESULTS}

For a simple validation of the network representations derived, the results obtained for the capacitive strip in a rectangular waveguide has been compared with the ones available in the technical literature. The transition from the parallel plate environment to the rectangular waveguide can, in fact, be effected very easily following the derivation presented

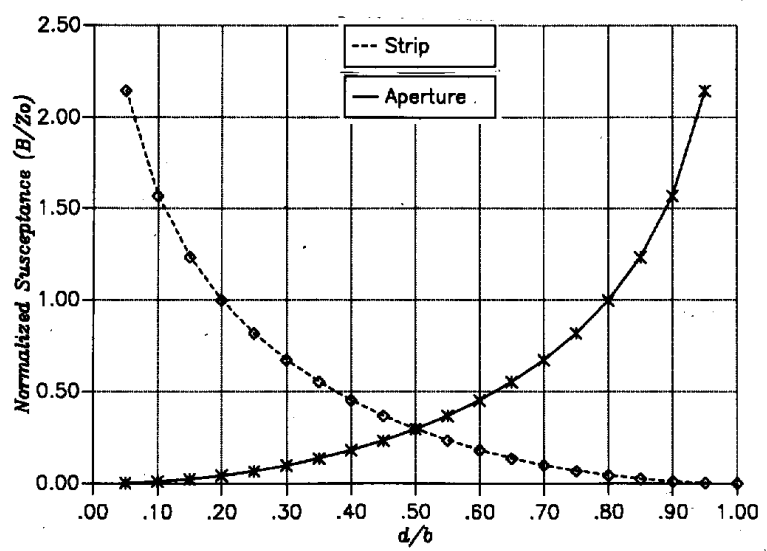

Fig. 6. Comparison of the normalized susceptance introduced by a centered capacitive strip and by a centered capacitive aperture in the lowest mode of a rectangular waveguide. The stars and squares indicate the results obtained using [2] while the continuous and the dashed line have been obtained using the network presented in this paper. The waveguide dimensions are $a=22.86 \mathrm{~mm}, b=10.16 \mathrm{~mm}$, and the frequency is $9.0 \mathrm{GHz}$. The parameter $d$ in this figure corresponds to $d 2-d 1$ in Fig. 1(a). 
in [5]. Fig. 6 shows the results of a comparison between [2] and our multimode network approach in the single mode case. The quantity computed is the normalized susceptance seen by the lowest mode as a function of the obstacle width for a centered capacitive strip and for a centered capacitive aperture. As we can see, the agreement is excellent.

A more detailed validation of the results obtained in this paper is included in a companion paper that focuses on the analysis of planar transmission line structures.

\section{APPLICATION}

As a simple application for the networks developed, we present in this section a procedure for the computation of the resonant frequencies of the cavity shown in Fig. 7. To analyze this structure, we consider propagation in $\hat{z}$ and the resonant frequencies are obtained by imposing the resonant condition of the resulting transverse equivalent network.

It is important to note that the networks derived apply to the case with $k_{x}=0$. We need, however, to consider the three different wave number, namely, $k_{x}, k_{y}$, and $k_{z}$. The derivation of the networks for the case $k_{x} \neq 0$ turns out to be a very simple problem (one only needs to substitute $k_{0}^{2}$ by $k_{0}^{2}-k_{x}^{2}$ in the old network representation) [5].

Following this procedure, the problem becomes extremely simple as the resulting modes are the hybrid H-type, and this kind of mode does not couple to E-type modes in the discontinuity. Furthermore, the waveguide is uniformly filled with a dielectric $\epsilon_{r}$, and this results in that no coupling between H-type and E-type modes is produced. The main implication of this is in that the structure of Fig. 7 can be studied under H-type and E-type excitation separately.

The transverse equivalent network for this structure under H-type excitation can be extracted from Fig. 2. In this figure we only need to add short circuits to both ends of the transmission lines representing the propagation of the modes. These short circuits take into account the effects of the metallic walls at the top and bottom of the box. Now the characteristic impedances of the transmission lines are the ones corresponding to H-type modes, namely,

$$
Z_{m, n}^{H}=\frac{\omega \mu_{0} k_{z, m, n}}{k_{0}^{2} \epsilon_{r}-k_{x}^{2}}
$$

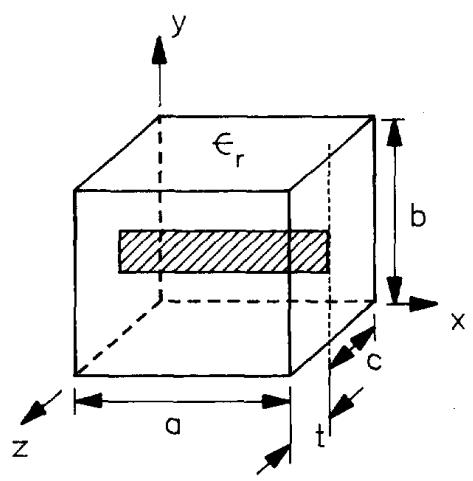

Fig. 7. Cavity whose resonant frequencies are computed using the network representation developed.
The frequency dependent constant $B$ becomes

$$
B_{m}^{H}=\frac{-j \pi \omega \mu_{0}}{b\left[k_{0}^{2} \epsilon_{r}-k_{x}^{2}\right]},
$$

the impedances in series attached to the network are

$$
\hat{Z}_{m, n}=\frac{-n}{2} B_{m}^{H}
$$

and, from the separability condition, we write straightforwardly

$$
k_{z, m, n}=\sqrt{k_{0}^{2} \epsilon_{r}-k_{y}^{2}-k_{x}^{2}} .
$$

In all the above equations, the expressions for the wave numbers in $\hat{x}$ and $\hat{y}$ directions are known to be $k_{x}=m \pi / a$, $k_{y}=n \pi / b$.

As an example, in the table below the resulting resonance frequencies are listed for a cavity with $a=15 \mathrm{~mm}, b=$ $10 \mathrm{~mm}, t=5 \mathrm{~mm}, c=5 \mathrm{~mm}$, and $\epsilon_{r}=2.2$. In the first column of the table, few resonant frequencies of the cavity without the discontinuity can be seen; whereas in the second column, the resonant frequencies of the structure with the metal strip are presented. In this table, $w$ is the width of the metal strip, and $p$ is the index for the wave number in $\hat{z}$. Furthermore, the indexes for the wave numbers in $\hat{x}$ and $\hat{y}$ have been fixed to $m=1$ and $n=1$, respectively.

TABLE I

\begin{tabular}{lcc}
\hline & $w=0$ & $w=1.2 \mathrm{~mm}$ \\
\hline$p=0$ & & $6.74 \mathrm{GHz}$ \\
$p=1$ & $15.81 \mathrm{GHz}$ & $15.81 \mathrm{GHz}$ \\
$p=2$ & $23.59 \mathrm{GHz}$ & $23.59 \mathrm{GHz}$ \\
$p=3$ & $32.68 \mathrm{GHz}$ & $32.68 \mathrm{GHz}$ \\
\hline
\end{tabular}

From the table, it can be seen that a new resonant frequency appears due to the presence of the metal strip; this technique can be applied to build resonators at lower frequencies without increasing the size of the box.

\section{CONCLUSION}

In this paper we have presented a set of novel, multimode equivalent network representations for a class of zerothickness capacitive discontinuities. The main features of the results obtained are that they are rigorous and that the results are given directly in terms of impedance or admittance matrix. Furthermore, the generic elements of the coupling matrix are derived in terms of integral equations that do not depend on frequency or absolute dimensions so that they need to be computed only once for each given geometry.

This last feature has very important consequences in terms of the numerical efficiency of codes based on this approach. In fact, the full formulation can be used to generate a set of coupling matrices corresponding to a discrete set of geometries. All other matrices corresponding to intermediate cases can then be simply obtained using an interpolation algorithm. 
As a result, the CPU time required for the characterization of the discontinuities becomes negligible. To obtain the electrical behavior of a structure over a given frequency range, one only needs to invert a system of linear equations (once for each frequency point). This feature can be exploited to produce codes that are computationally very efficient.

In addition to the theoretical formulations, we have presented in this paper various results, with the aim of validating the multimode network representation derived. All the results included indicate very good behavior both in terms of numerical and of modal convergency.

The main application of the results obtained is to the study of planar transmission line structures. The networks developed can in fact be used to study planar transmission line geometries by using the transverse resonance technique [9]. Single and coupled lines can be easily studied. In addition, several discontinuities can be easily cascaded, thereby expanding the range of applicability of this approach to a very large variety of structures.

\section{APPENDIX A}

To obtain the explicit expressions in (17), we first substitute the expression for the vector mode functions in (14), obtaining

$$
\begin{aligned}
\sqrt{\frac{\epsilon_{m}}{2}} \cos \frac{m \pi}{b} y= & \int_{d_{1}}^{d_{2}} M_{m}\left(y^{\prime}\right) \frac{B}{\left(\epsilon_{r}^{(1)}+\epsilon_{r}^{(2)}\right)} \\
& \cdot \sum_{n=1}^{\infty} n \cos \left(\frac{n \pi}{b} y\right) \cos \left(\frac{n \pi}{b} y^{\prime}\right) d y^{\prime}
\end{aligned}
$$

where we have chosen $A_{o}$ such that

$$
A_{o}\left(-y_{0}\right) \sqrt{\frac{2}{b}}=1
$$

We then integrate ( 91 ) by parts, obtaining

$$
\begin{aligned}
\sqrt{\frac{\epsilon_{m}}{2}} \cos \frac{m \pi}{b} y= & -\int_{d_{1}}^{d_{2}} M_{m}^{\prime}\left(y^{\prime}\right) \frac{B b}{\pi\left(\epsilon_{r}^{(1)}+\epsilon_{r}^{(2)}\right)} \\
& \cdot \sum_{n=1}^{\infty} \cos \left(\frac{n \pi}{b} y\right) \sin \left(\frac{n \pi}{b} y^{\prime}\right) d y^{\prime}
\end{aligned}
$$

where $M_{m}^{\prime}$ is the derivative of $M_{m}$, and we have imposed the condition that $M_{m}(y)=0$ for $y=d_{1}$ and $y=d_{2}$ (this condition will be reintroduced later in a self-consistent fashion).

The kernel of (93) can now be summed using the relation ([1], Ch. 6.2.2)

$$
\begin{aligned}
& \lim _{\delta \rightarrow \infty} \sum_{n=1}^{\infty} e^{-\delta n} \cos \left(\frac{n \pi}{b} y\right) \sin \left(\frac{n \pi}{b} y^{\prime}\right)= \\
& \frac{1}{2} \frac{\sin \left(\frac{\pi y^{\prime}}{b}\right)}{\cos \left(\frac{\pi y}{b}\right)-\cos \left(\frac{\pi y^{\prime}}{b}\right)}
\end{aligned}
$$

so that (93) becomes

$$
\begin{aligned}
\sqrt{\frac{\epsilon_{m}}{2}} f_{m}(y)= & -\int_{d_{1}}^{d_{2}} M_{m}^{\prime}\left(y^{\prime}\right) \frac{B b}{2 \pi\left(\epsilon_{r}^{(1)}+\epsilon_{r}^{(2)}\right)} \\
& \cdot \frac{\sin \left(\frac{\pi y^{\prime}}{b}\right)}{\cos \left(\frac{\pi y}{b}\right)-\cos \left(\frac{\pi y^{\prime}}{b}\right)} d y^{\prime}
\end{aligned}
$$

where

$$
f_{m}(y)=\cos \left(\frac{m \pi}{b} y\right) .
$$

It is now convenient to use the changes of variable $\cos \left(\pi y^{\prime} / b\right)=\eta^{\prime}$ and $\eta=k_{1}+k_{2} \nu$, where $k_{1}$ and $k_{2}$ are defined in (24), so that (95) becomes

$$
\sqrt{\frac{\epsilon_{m}}{2}} f_{m}\left(\frac{b}{\pi} \arccos \left[k_{1}+k_{2} \nu\right]\right)=\frac{1}{\pi} \int_{-1}^{1} \frac{F_{m}\left(\nu^{\prime}\right)}{\nu^{\prime}-\nu} d \nu^{\prime}
$$

where

$$
\frac{-B b^{2}}{2 \pi\left(\epsilon_{r}^{(1)}+\epsilon_{r}^{(2)}\right)} M_{m}^{\prime}\left(\frac{b}{\pi} \cos \left[k_{1}+k_{2} \nu^{\prime}\right]\right)=F_{m}\left(\nu^{\prime}\right)
$$

and $k_{1}$ and $k_{2}$ are defined in (24). Equation (97) is now a standard Cauchy-type singular integral equation of known solution [7], namely

$$
\begin{aligned}
F_{m}(\nu)= & \sqrt{\frac{\epsilon_{m}}{2}} \frac{1}{\sqrt{1-\nu^{2}}}\left[C_{m}-\frac{1}{\pi} \int_{-1}^{1} \frac{\sqrt{1-\nu^{\prime 2}}}{\nu^{\prime}-\nu}\right. \\
& \left.\cdot f_{m}\left(\frac{b}{\pi} \arccos \left[k_{1}+k_{2} \nu^{\prime}\right]\right) d \nu^{\prime}\right]
\end{aligned}
$$

It is now convenient to define $Q_{m}(\nu)$ as in (20) so that (99) becomes

$$
F_{m}(\nu)=\sqrt{\frac{\epsilon_{m}}{2}} \frac{1}{\sqrt{1-\nu^{2}}}\left[C_{m}-Q_{m}(\nu)\right] .
$$

We next derive the expression for $Y_{n, m}$ in (17). To do so, we introduce in (16) the expression for the vector mode functions, obtaining

$$
Y_{n, m}=\sqrt{\frac{\epsilon_{n}}{2}} \int_{d_{1}}^{d_{2}} M_{m}(y) \cos \left(\frac{n \pi}{b} y\right) d y
$$

where we have used for $A_{o}$ the expression in (92).

Let us now recall that

$$
M_{m}(y)=\int_{d_{1}}^{y} M_{m}^{\prime}\left(y^{\prime}\right) d y^{\prime} .
$$

Using (98) and (100), eq. (102) is transformed into

$$
\begin{gathered}
M_{m}\left(\frac{b}{\pi} \arccos \left[k_{1}+k_{2} \nu\right]\right)=\frac{2 k_{2}}{B_{b}} \sqrt{\frac{\epsilon_{m}}{2}}\left(\epsilon_{r}^{(1)}+\epsilon_{r}^{(2)}\right) \\
\cdot \int_{-1}^{\nu} \frac{\left(C_{m}-Q_{m}\left(\nu^{\prime}\right)\right)}{\sqrt{1-\nu^{\prime}} \sqrt{1-\left(k_{1}+k_{2} \nu^{\prime}\right)^{2}}} d \nu^{\prime} .
\end{gathered}
$$


Now, using the above expression, we can rewrite (101) in the form

$$
\begin{aligned}
Y_{n, m}= & \sqrt{\epsilon_{n} \epsilon_{m}} \frac{-k_{2}^{2}}{B \pi}\left(\epsilon_{r}^{(1)}+\epsilon_{r}^{(2)}\right) \\
& \cdot \int_{-1}^{1} \frac{\left(C_{m}-Q_{m}\left(\nu^{\prime}\right)\right)}{\sqrt{1-\nu^{\prime 2}} \sqrt{1-\left(k_{1}+k_{2} \nu^{\prime}\right)^{2}}} \\
& \cdot \int_{\nu^{\prime}}^{1} \frac{\cos \left(n \arccos \left[k_{1}+k_{2} \nu\right]\right)}{\sqrt{1-\left(k_{1}+k_{2} \nu\right)^{2}}} d \nu d \nu^{\prime}
\end{aligned}
$$

where we have inverted the order of the integrations. At this point, the integral in $d \nu$ can be evaluated analytically, so that we obtain the expressions given in (18).

The last analytical effort is the computation of constant $C_{m}$. To find its value, we use (103), and the condition that $M_{m}(y)=0$ for $y=d_{1}$ and $y=d_{2}$, obtaining

$$
\begin{aligned}
0= & \frac{2 K_{2}}{B b}\left(\epsilon_{r}^{(1)}+\epsilon_{r}^{(2)}\right) \sqrt{\frac{\epsilon_{m}}{2}} \\
& \cdot \int_{-1}^{1} \frac{\left(C_{m}-Q_{m}\left(\nu^{\prime}\right)\right)}{\sqrt{1-\left(k_{1}+k_{2} \nu^{\prime}\right)^{2}} \sqrt{1-\nu^{\prime 2}}} d \nu^{\prime}
\end{aligned}
$$

from which $C_{m}$ can be found in the form shown in (22) and (23).

For the solution of the double strip, an additional gap must be added to the integration intervals of the relevant integrals, thereby obtaining (26) and (27). Equation (27) is again a standard Cauchy-type singular integral equation with one gap inside the integration interval. The solution for this equation can be found in ([1], Ch. 7.1.2), namely,

$$
F_{m}(\xi)=\frac{1}{X(\xi)}\left[P_{m}(\xi)-\frac{1}{\pi} f_{-1}^{1} \frac{X\left(\xi^{\prime}\right) f_{m}\left(\xi^{\prime}\right)}{\xi^{\prime}-\xi} d \xi^{\prime}\right] \sqrt{\frac{\epsilon_{m}}{2}}
$$

where $P_{m}(\xi), X(\xi)$, and $f_{m}(\xi)$ are those defined in (38), (31), and (45), respectively. Following a procedure similar to the one used for the single strip, (26) now becomes

$$
\begin{aligned}
Y_{n, m}= & \frac{\sqrt{\epsilon_{n} \epsilon_{m}}}{\pi B}\left(\epsilon_{r}^{(1)}+\epsilon_{r}^{(2)}\right) \\
& \cdot f_{-1}^{1} \frac{\left[Q_{m}\left(\xi^{\prime}\right)-P_{m}\left(\xi^{\prime}\right)\right] R_{n}\left(\xi^{\prime}\right)}{X\left(\xi^{\prime}\right) \sqrt{1-\left(k_{1}+k_{2} \xi^{\prime}\right)^{2}}} d \xi^{\prime}
\end{aligned}
$$

where $R_{n}$ is defined in (30), and $Q_{m}$ takes the form

$$
Q_{m}(\xi)=\frac{1}{\pi} f_{-1}^{1} \frac{X\left(\xi^{\prime}\right) f_{m}\left(\xi^{\prime}\right)}{\xi^{\prime}-\xi} d \xi^{\prime}
$$

At this point, it is convenient to introduce the following changes of variables:

$$
\xi_{u, l}=k_{1}^{u, l}+k_{2}^{u, l} \nu
$$

where $k_{1}^{u}, k_{2}^{u}, k_{1}^{l}$, and $k_{2}^{l}$ are found in (36) and (37). Using these changes of variables for both $\xi$ and $\xi^{\prime}$, (108) splits in the four shown in (43) and (44). If the same change of variable is used for the $\xi$ variable in (107), one can easily obtain (28) and (29) for the coupling matrix elements.
The completion of the solution for the double strip is obtained by finding the two unknown constants appearing in $P_{m}$ in (38). Using the same procedure as for the single strip problem, (103) now takes the form

$$
\begin{array}{r}
M_{m}\left(\frac{b}{\pi} \arccos \left[k_{1}+k_{2} \xi\right]\right)=\frac{2 k_{2}}{B b}\left(\epsilon_{r}^{(1)}+\epsilon_{r}^{(2)}\right) \frac{\sqrt{\epsilon_{m}}}{2} \\
\cdot f_{-1}^{\xi} \frac{P_{m}\left(\xi^{\prime}\right)-Q_{m}\left(\xi^{\prime}\right)}{X\left(\xi^{\prime}\right) \sqrt{1-\left(k_{1}+k_{2} \xi^{\prime}\right)^{2}}} d \xi^{\prime}
\end{array}
$$

Now, imposing the condition $M_{m}(y)=0$ for $y=d_{1}^{(1)}$, $y=d_{1}^{(2)}, y=d_{2}^{(1)}$, and $y=d_{2}^{(2)}$, the following two relations are obtained:

$$
\begin{aligned}
& 0=\int_{-1}^{\xi_{2}^{(1)}} \frac{P_{m}\left(\xi^{\prime}\right)-Q_{m}\left(\xi^{\prime}\right)}{X\left(\xi^{\prime}\right) \sqrt{1-\left(k_{1}+k_{2} \xi^{\prime}\right)^{2}}} d \xi^{\prime} \\
& 0=\int_{\xi_{1}^{(2)}}^{2 l-1} \frac{P_{m}\left(\xi^{\prime}\right)-Q_{m}\left(\xi^{\prime}\right)}{X\left(\xi^{\prime}\right) \sqrt{1-\left(k_{1}+k_{2} \xi^{\prime}\right)^{2}}} d \xi^{\prime}
\end{aligned}
$$

where $\xi_{1}^{(2)}$ and $\xi_{2}^{(1)}$ are those defined in (35). It is now convenient to use the changes of variables (109) in (111) and (112), so that after defining (41) and (42), the unknown constants take the form shown in (39) and (40).

\section{APPENDIX B}

To obtain the final results given in (59), we substitute the expression for the vector mode functions in (56), obtaining

$$
\begin{aligned}
\sqrt{\frac{\epsilon_{m}}{2}} \cos \frac{m \pi}{b} y= & \int_{d_{1}}^{d_{2}} M_{m}\left(y^{\prime}\right) B^{-1}\left(\epsilon_{r}^{(1)}+\epsilon_{r}^{(2)}\right) \\
& \cdot \sum_{n=1}^{\infty} \frac{1}{n} \cos \left(\frac{n \pi}{b} y\right) \cos \left(\frac{n \pi}{b} y^{\prime}\right) d y^{\prime}
\end{aligned}
$$

where we have chosen $A_{o}$ such that

$$
A_{o}\left(-x_{0}\right) \sqrt{\frac{2}{b}}=1 \text {. }
$$

The kernel of (113) can be summed using the relation ([1], Ch. 6.1.2)

$$
\begin{aligned}
& \sum_{n=1}^{\infty} \frac{1}{n} \cos \left(\frac{n \pi}{b} y\right) \cos \left(\frac{n \pi}{b} y^{\prime}\right)=-\frac{1}{2} \log \\
& \cdot\left[2\left|\cos \frac{\pi y}{b}-\cos \frac{\pi y^{\prime}}{b}\right|\right] .
\end{aligned}
$$

Now, using the following change of variable, originally due to Schwinger [10]:

$$
\cos \left(\frac{\pi}{b} y\right)=C+S \cos x
$$

(113) becomes

$f_{m}(x)=\int_{0}^{\pi} F_{m}\left(x^{\prime}\right)\left[-\frac{1}{2} \log S+\sum_{k=1}^{\infty} \cos (k x) \cos \left(k x^{\prime}\right)\right] d x^{\prime}$ 
where we have changed the index of the summation from $n$ to $k$ for convenience, and

$$
\begin{array}{r}
f_{m}(x)=\sqrt{\frac{\epsilon_{m}}{2}} \cos [m \arccos (C+S \cos x)] \\
F_{m}(x)=\frac{M_{m}\left[\frac{b}{\pi} \arccos (C+S \cos x)\right]}{\left(-\frac{\pi}{b}\right) \sqrt{1-(C+S \cos x)^{2}}} \\
\cdot B^{-1}\left(\epsilon_{r}^{(1)}+\epsilon_{r}^{(2)}\right)(-S) \sin x .
\end{array}
$$

(In the above expressions, $C$ and $S$ are defined as in (61) and (62).)

The set of functions $\cos (k x)$ is a complete, orthogonal set of functions between 0 and $\pi$, so it is possible to express any function in the form

$$
f_{m}(x)=C_{0}^{(m)}+\sum_{k=1}^{\infty} C_{k}^{(m)} \cos (k x)
$$

where the coefficients can be easily obtained using the orthogonality property, yielding

$$
\begin{aligned}
& C_{0}^{(m)}=\frac{1}{\pi} \sqrt{\frac{\epsilon_{m}}{2}} D_{0}^{(m)} \\
& C_{k}^{(m)}=\frac{2}{\pi} \sqrt{\frac{\epsilon_{m}}{2}} D_{k}^{(m)}
\end{aligned}
$$

and where $D_{0}^{(m)}$ and $D_{k}^{(m)}$ are those defined in (60). Comparing (117) and (120), we then obtain

$$
\begin{gathered}
\int_{0}^{\pi} F_{m}\left(x^{\prime}\right) d x^{\prime}=\frac{-2}{\log S} \frac{1}{\pi} \sqrt{\frac{\epsilon_{m}}{2}} D_{0}^{(m)} \\
\int_{0}^{\pi} F_{m}\left(x^{\prime}\right) \cos \left(k x^{\prime}\right) d x^{\prime}=k \frac{2}{\pi} \sqrt{\frac{\epsilon_{m}}{2}} D_{k}^{(m)} .
\end{gathered}
$$

The function $F_{m}(x)$ can now be expressed as a linear combination of the same set of functions, namely $\cos (k x)$, obtaining

$$
F_{m}=\sum_{k=0}^{\infty} E_{k}^{(m)} \cos (k x) .
$$

Using again the orthogonality property, we find

$$
\begin{gathered}
E_{0}^{(m)}=\frac{1}{\pi} \int_{0}^{\pi} F_{m}(x) d x \\
E_{k}^{(m)}=\frac{2}{\pi} \int_{0}^{\pi} F_{m}(x) \cos (k x) d x .
\end{gathered}
$$

From (123) and (124), we can now write the general expression for $E_{k}^{(m)}$, namely,

$$
\begin{gathered}
E_{0}^{(m)}=\left(\frac{1}{\pi}\right)^{2}\left(\frac{-2}{\log S}\right) \sqrt{\frac{\epsilon_{m}}{2}} D_{0}^{(m)} \\
E_{k}^{(m)}=\left(\frac{2}{\pi}\right)^{2} k \sqrt{\frac{\epsilon_{m}}{2}} D_{k}^{(m)}
\end{gathered}
$$

So that $F_{m}(x)$ is found to be

$$
\begin{aligned}
F_{m}(x)= & \left(\frac{1}{\pi}\right)^{2}\left(\frac{-2}{\log S}\right) \sqrt{\frac{\epsilon_{m}}{2}} D_{0}^{(m)} \\
& +\sum_{k=1}^{\infty}\left(\frac{2}{\pi}\right)^{2} k \sqrt{\frac{\epsilon_{m}}{2}} D_{k}^{(m)} \cos (k x)
\end{aligned}
$$

We next derive the expression for $Z_{n, m}$ in (59). To do so, we introduce in (58) the expression for the vector mode function, obtaining

$$
Z_{n, m}=\sqrt{\frac{\epsilon_{n}}{2}} \int_{d_{1}}^{d_{2}} M_{m}(y) \cos \left(\frac{n \pi}{b} y\right) d y
$$

where we have used for $A_{o}$ the expression in (114). Using (119) and (130), eq. (131) takes the form shown in (59), with the coefficients $D_{k}^{(m)}$ defined as in (60).

For the double gap, the only difference is that in (113) and (131), there is an additional gap in the integrals. To analytically solve the resulting equations, it is convenient to define the following auxiliary functions:

$$
\begin{gathered}
\Phi_{m}\left(y^{\prime}\right)= \begin{cases}M_{m}\left(y^{\prime}\right) ; & \forall y^{\prime} \notin\left[d_{2}^{(1)}, d_{1}^{(2)}\right] \\
0 ; & \forall y^{\prime} \in\left[d_{2}^{(1)}, d_{1}^{(2)}\right]\end{cases} \\
H_{m}(y)= \begin{cases}f_{m}(y) ; & \forall y^{\prime} \notin\left[d_{2}^{(1)}, d_{1}^{(2)}\right] \\
f_{m}(y)+g_{m}(y) ; & \forall y^{\prime} \in\left[d_{2}^{(1)}, d_{1}^{(2)}\right]\end{cases}
\end{gathered}
$$

where $f_{m}(y)$ is defined in (118), and $g_{m}(y)$ is an unknown function. A similar procedure was also used in ([1], Ch. 7.1.2), but with a different definition of the auxiliary functions.

On using (132) and (133), we remove the gap from the integrals in (113) and (116), yielding

$$
\begin{aligned}
H_{m}(y)= & \int_{d_{1}^{(1)}}^{d_{2}^{(2)}} \Phi_{m}\left(y^{\prime}\right) B^{-1}\left(\epsilon_{r}^{(1)}+\epsilon_{r}^{(2)}\right) \\
& \cdot \sum_{n=1}^{\infty} \frac{1}{n} \cos \left(\frac{n \pi}{b} y\right) \cos \left(\frac{n \pi}{b} y^{\prime}\right) d y^{\prime} \\
Z_{n, m}= & \sqrt{\frac{\epsilon_{m}}{2}} \int_{d_{1}^{(1)}}^{d_{2}^{(2)}} \Phi_{m}(y) \cos \left(\frac{n \pi}{b} y\right) d y
\end{aligned}
$$

Following a procedure similar to the one previously outlined for the single aperture, (134) is now transformed into

$$
\sum_{k=1}^{\infty} 2 k I_{k}^{(m)} \cos (k x)=\int_{x_{1}}^{x_{2}} g_{m}\left(x^{\prime}\right) 2 \sum_{l=1}^{\infty} l \cos \left(l x^{\prime}\right) \cos (l x) d x^{\prime}
$$

where the coefficient $I_{k}^{(m)}$ is found in (67), and $x_{1}, x_{2}$ are those defined in (85) and (86), respectively.

Equation (136) can be reduced to a Cauchy-type singular integral equation for the unknown function $g_{m}(x)$. Its solution was reported in Appendix A.

Once $g_{m}(x)$ is found, the generic expression for the coupling matrix elements in (65) can be obtained in the form shown in Section V.

\section{REFERENCES}

[1] L. Lewin, Theory of Waveguides. New York: Wiley, 1974

[2] N. Marcuvitz, Waveguide Handbook, Vol. 10, MIT Radiation Laboratory Series. New York: McGraw-Hill, 1951, p. 221.

[3] T.E. Rozzi, "Network modeling of interacting capacitive irises and steps in waveguide," in Proc. IEEE Microwave Service to Man Microwave Symp., Palo Alto, CA, May 12-14, 1975, pp. 197-199.

[4] M. Guglielmi and C. Newport, "Rigorous, multimode equivalent network representation of inductive discontinuities," IEEE Trans. Microwave Theory Tech., vol. MTT-38, pp. 1651-1659, Nov. 1990. 
[5] H.M. Altshuler and L. O. Goldstone, "On network representations of certain obstacles in waveguide regions," IRE Trans. Microwave Theory Tech., Apr. 1959, pp. 213-221.

[6] N. Marcuvitz, Waveguide Handboak, Vol. 10, MIT Radiation Laboratory Series. New York: McGraw-Hill, 1951, p. 62

[7] F.G. Tricomi, Integral Equations. New York: Interscience, 1957 pp. 166-197.

[8] M. Abramowitz and I, A. Stegun Editors, Handbook of Mathematical Functions. New York: Dover, 1972, p. 919.

[9] T. Itoh, ED., Numerical Techniques for Microwave and Millimeter-Wave Passive Structures. New York: Wiley, 1989, pp. 654-664.

[10] J. Schwinger and D. S. Saxon, Discontinuities in Waveguides, Notes on Lectures by Julian Schwinger: New York: Gordon and Breach Science, 1968 , p. 72.

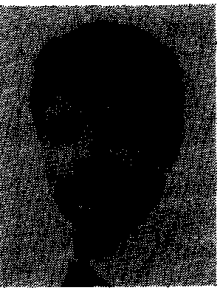

Alejandro Alvarez Melcón was born in Madrid, Spain, in 1965. He received the Ingeniero degree in telecommunication engineering from the Polytechnic University of Madrid (UPM), in 1991.

In 1988 he joined the Signal, System and Radiocommunication Department of UPM as a Research Student, where he was involved in the design, testing, and measurement of broadband spiral antennas for EMS equipment. Since 1991 he has been working in the Microwave Systems Department of the European Space Agency (ESA/ESTEC), where he is currently involved in the development of analytical and numerical tools for the analysis of discontinuities in waveguides and microwave filter designs.

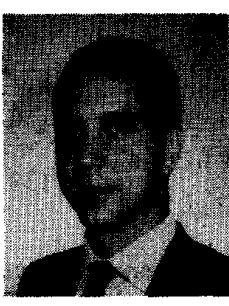

Marco Guglielmi was born in Rome, Italy, on December 17, 1954. He received the degree "Laurea in Ingegneria Elettronica" in 1979 from the University of Rome "La Sapieza," Rome, Italy, where in 1980 he also attended the "Scuola di Specializzazione in Elettromagnetismo Applicato." In 1981 he was awarded a Fulbright Scholarship in Rome, Italy, and an HISP scholarship (Halsey International Scholarship Program) from the University of Bridgeport, Bridgeport, CT, where he received the M.S. degree in electrical engineering in 1982. He received the Ph.D. degree in Electrophysics from Polytechnic University, Brooklyn, NY, in 1986.

From 1984 to 1986 he was Academic Associate at Polytechnic University, and from 1986 to 1988 he was Assistant Professor in the same institution. From 1988 to 1989 he was Assistant Professor at the New Jersey Institute of Technology, Newark. In 1989 he joined the RF System Division of the European Space Research and Technology Centre, Noordwijk, The Netherlands, where he is currently involved in the development of passive microwave components for space applications. His professional interests include the areas of solid-state devices and circuits, periodic structures, phased arrays and millimeter-wave leaky-wave antennas, network representations of waveguide discontinuities, and microwave filtering structures. 